\title{
Beta burns following radionuclide synovectomy
}

\author{
Marek Marcin Chojnowski ${ }^{1}$, Maria Teresa Płazińska², Marek Sławomir Chojnowski ${ }^{3}$, Leszek Królicki \\ ${ }^{1}$ Nuclear Medicine Department, Central Clinical Hospital, Warsaw, Poland \\ ${ }^{2}$ Nuclear Medicine Department, Warsaw Medical University, Poland \\ ${ }^{3}$ Clinical Department of General and Colorectal Surgery, Bielański Hospital, Warsaw, Poland
}

\begin{abstract}
Radionuclide synovectomy (RSV) is a form of minimally invasive treatment of persistent joint in flammation. The procedure has a high safety profile and the occurrence of serious adverse events, such as full-thickness skin radiation necrosis, is rare. Less severe radiation events, while more common, are usually benign and self-limiting.

We present two cases of low-grade beta burns that developed after RSV, despite proper injection technique. The potential long-term risk of such exposure is also discussed, with reference to historical radiation incidents. While low-grade beta burns after RSV usually pose little danger to the patient, any clinician involved in radionuclide treatment of arthritis should be aware of their existence and management
\end{abstract}

Key words: rheumatoid arthritis, radiosynovectomy, osteoarthrosis, beta burns.

\section{Introduction}

Radionuclide synovectomy (RSV) is a form of minimally invasive treatment of persistent joint inflammation. It involves intra-articular injection of a $\beta$-emitting radiopharmaceutical with subsequent irradiation and necrosis of hypertrophic synovial tissue [1]. The procedure has a high safety profile, but as in any medical intervention, a small percentage of patients may experience serious adverse events. The most dangerous complication is radioisotope leakage resulting in full-thickness skin necrosis; however, its incidence is extremely low [2-4]. Milder radiation events, usually benign and transient in nature, are more common. Physicians dealing with inflammatory joint diseases and radioisotope treatment should be aware of such complications, their management and prognosis. We present two cases of patients who experienced mild to moderate irradiation of extraarticular tissues after RSV.

\section{Case 1}

The first patient is a 36-year-old woman with oneyear history of rheumatoid arthritis. She reported painful swelling of the right wrist joint, which was refractory to methotrexate. The ultrasound (US) examination detected synovial hypertrophy with effusion in the right wrist. Due to an unsatisfactory response to diseasemodifying antirheumatic drugs, the patient was qualified for RSV.

Using direct US guidance, 37 megabecquerels ( $\mathrm{MBq}$ ) of colloidal rhenium-186 ( $\left.{ }^{186} \mathrm{Re}\right)$ sulfide were injected into the right radio-carpal joint. After immobilizing the right hand with sterile dressing, post-therapeutic arthroscintigraphy was performed in the palmo-dorsal projection (Nucline $\mathrm{TH}-22$ single-head gamma camera, Mediso). The scintigram showed a uniform distribution of the radiopharmaceutical in the radio-carpal joint, and another area of mild radioactivity in the radial aspect of the hand (Fig. 1A). This extra-articular activity was initially considered a spilled drop of the radiopharmaceutical and thus the skin was thoroughly decontaminated, according to standard procedures.

During a routine follow-up after 4 weeks, the patient reported partial reduction of pain and swelling of the right wrist joint. The clinical examination, however, revealed a light-brown patch on the skin, along the tendon of the abductor pollicis longus muscle (Figs. 1B, 1C). The

\footnotetext{
Address for correspondence:

Marek Marcin Chojnowski, Nuclear Medicine Department, Central Clinical Hospital, 1A Banacha St., 02-097 Warsaw, Poland,

e-mail: marekchojnowski84@gmail.com

Submitted: 20.03.2018; Accepted: 7.05.2018
} 
A

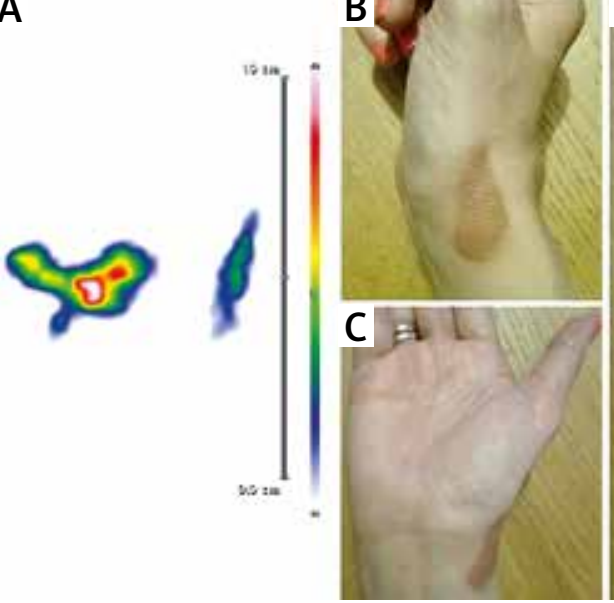

D

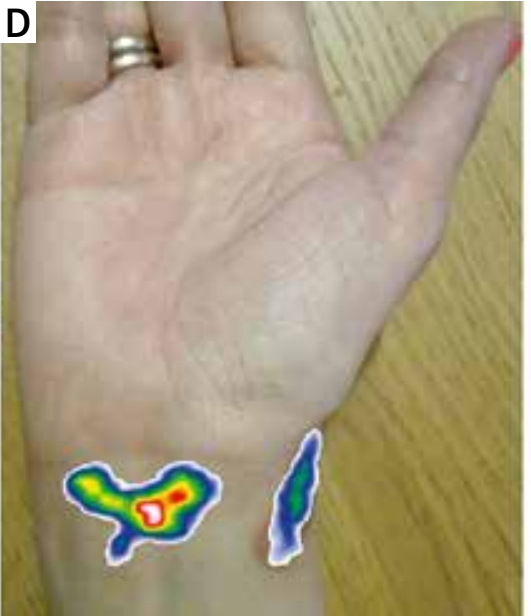

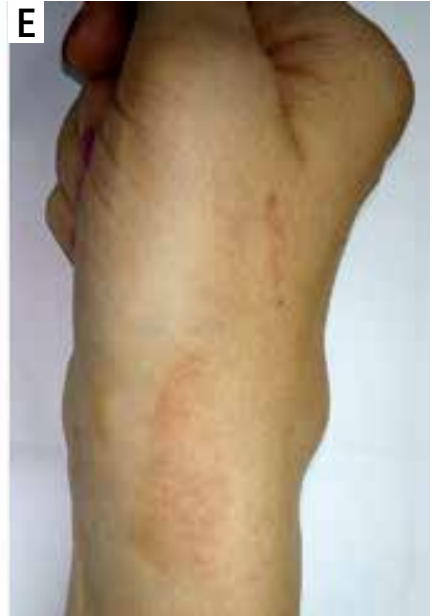

Fig. 1. Post-therapeutic ${ }^{186}$ Re arthroscintigraphy of the wrist (palmo-dorsal projection) (A). Beta burns four weeks after RSV (B, C). Scintigram superimposed on the skin lesion (D). Beta burns six months after RSV (E).

patient did not experience pain, tenderness or any other unpleasant sensation in this area. When compared with the post-therapeutic scintigram, the skin lesion corresponded to the area of extra-articular radioactivity (Fig. 1D). The skin lesion was diagnosed as a low-grade radiodermatitis, specifically, a mild beta burn. Since the brown skin patch was the sole symptom, a watchful waiting approach was chosen. During a 6-month follow-up, no other complications emerged, the lesion steadily waned, and only slight skin discoloration remained (Fig. 1E). Moreover, the symptoms of right wrist joint inflammation subsided.

The most likely explanation of this phenomenon is a radiopharmaceutical leakage to the first extensor tendon compartment from the joint cavity. Such communication could be a pre-existing condition (possibly an anatomical variant) or a result of synovial membrane erosive activity in course of chronic inflammation. From inside the tendon sheath, not shielded by bones of the joint or the articular capsule, the $\beta$-particles can easily irradiate and damage adjacent soft tissues. Since ${ }^{186} \mathrm{Re}$ radiation has a small tissue penetration range (about $1.2 \mathrm{~mm}$ ) and a moderate energy (0.98 MeV) [5], beta burns associated with ${ }^{186}$ Re radiopharmaceuticals are usually benign and require no treatment, as in the present case.

\section{Case 2}

The second patient is an 82-year-old woman with knee osteoarthritis (OA) and no history of systemic inflammatory joint disease. In the right knee joint, apart from typical pain and limited mobility, the patient also suffered from recurrent effusion secondary to OA. The effusion was refractory to repeated joint punctures with intra-articular glucocorticoid administration. The patient did not consent to knee arthroplasty or arthroscopic joint debridement and, since she suffered from type 2 diabetes, numerous glucocorticoid injections made the patient's blood glucose level difficult to control. Taking into consideration all available therapeutic options, the patient was referred for RSV as a supportive treatment.

Under US guidance, the suprapatellar recess of the right knee joint was punctured from the lateral approach. Approximately $40 \mathrm{ml}$ of clear, yellowish fluid was evacuated, and $185 \mathrm{MBq}$ of colloidal yttrium-90 $\left({ }^{90} \mathrm{Y}\right)$ citrate was injected followed by $2 \mathrm{ml}$ of sterile saline to flush the residual radiopharmaceutical from the needle. A sterile dressing was applied, the knee was immobilized, and the patient was advised not to walk or stand for at least 48 hours in order to minimize the risk of radioactive leakage. Thirty minutes after RSV, post-therapeutic positron emission tomography/computed tomography (PET/CT) with ${ }^{90} Y$ was performed (Biograph 64, Siemens) [6], showing diffuse distribution of the radiopharmaceutical in the suprapatellar recess and medial knee compartment, with no evident extra-articular radioactivity (Fig. 2).

During the follow-up visit after 4 weeks, the patient complained of a draining sinus with discharge of yellowish, turbid fluid and tender erythema at the site of the joint puncture (Figs. 3A, 3B). US examination revealed that the sinus canal was $8 \mathrm{~mm}$ deep and was surrounded by hyperechoic tissue, with an increased power Doppler signal. Deeper layers of subcutaneous tissue were within normal limits, the articular capsule was intact and the wound did not communicate with the joint cavity. The patient admitted that she had not complied with the recommended knee immobilization and bed rest after RSV. Accordingly, the sinus was most likely the result of a par- 

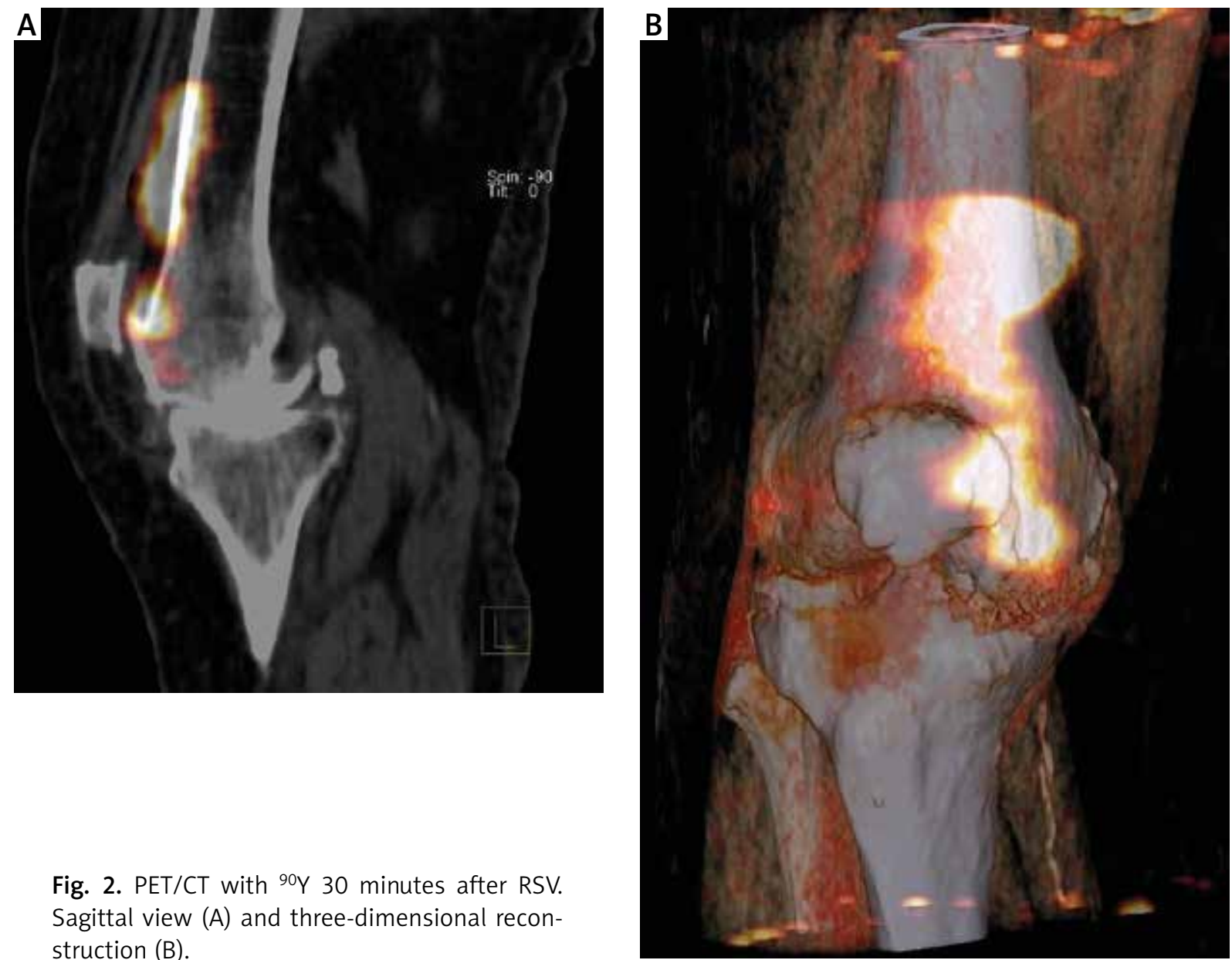

Fig. 2. PET/CT with ${ }^{90} \mathrm{Y} 30$ minutes after RSV. Sagittal view $(A)$ and three-dimensional reconstruction (B).

tial radiopharmaceutical reflux along the needle tract into the subcutaneous tissue.

Microbiological cultures taken from the wound were negative. The consulting surgeon advised against surgical excision of the lesion, since poorly controlled diabetes and obesity could compromise the wound healing. A conservative approach was employed, including flushing the sinus with saline, debridement of necrotic tissue, application of hydrocortisone/oxytetracycline ointment and regular changes of the sterile dressing. Six months after the RSV, the fluid discharge and tenderness subsided, leaving a depression in the skin with scar tissue (Fig. 3C).

\section{Discussion}

Full-thickness skin necrosis after RSV is extremely rare [4]. Appropriate injection technique and detailed instructions given to the patient allow such complications to be prevented altogether, and indeed in some centers such events do not occur [7]. In our department, in a series of $>2000$ joints treated within 5 years we have ob-

served four cases of mild beta burns (two are presented in detail in this article, and two are shown in Figs. 4A-4C) and one case of superficial skin necrosis, which healed within 8 months without hyperbaric oxygen treatment or surgical intervention (Fig. 4D). However, there are no statistics regarding the significance of such radiation sequelae and their long-term consequences, especially the risk of skin malignancy.

Some data may be drawn from experience with external beam radiotherapy (EBRT), where skin radiation burns are not uncommon. For example, hyperbaric oxygen treatment, which is an effective therapy for EBRT burns [8], can be successfully employed in managing RSV complications [4]. However, the type and energy of ionizing radiation differ significantly between EBRT and RSV, with high-energy (up to $25 \mathrm{MeV}$ ) [9], high-penetration $\mathrm{X}$-rays in the first, and lower energy (up to $2.2 \mathrm{MeV}$ ) [5], low-penetration $\beta$-particles in the latter. Hence, one should be careful when drawing conclusions about longterm sequelae of RSV based on experience with EBRT. 


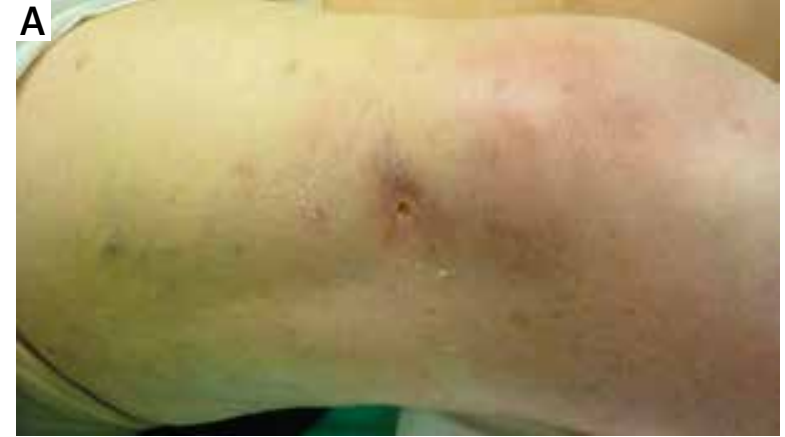

C

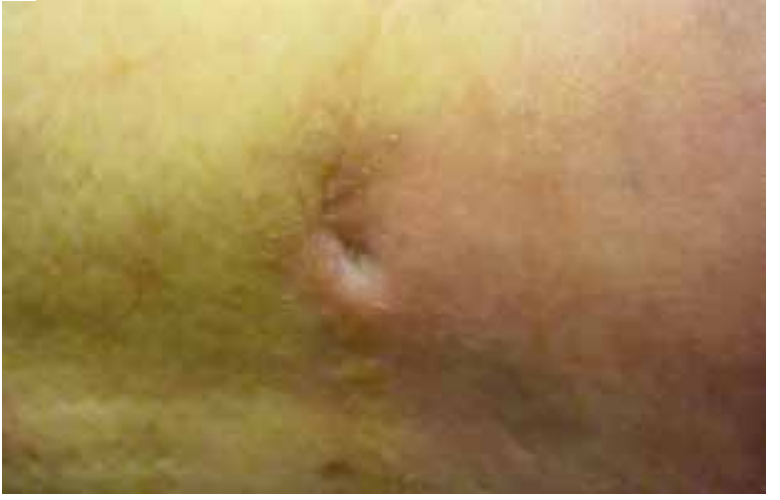

A

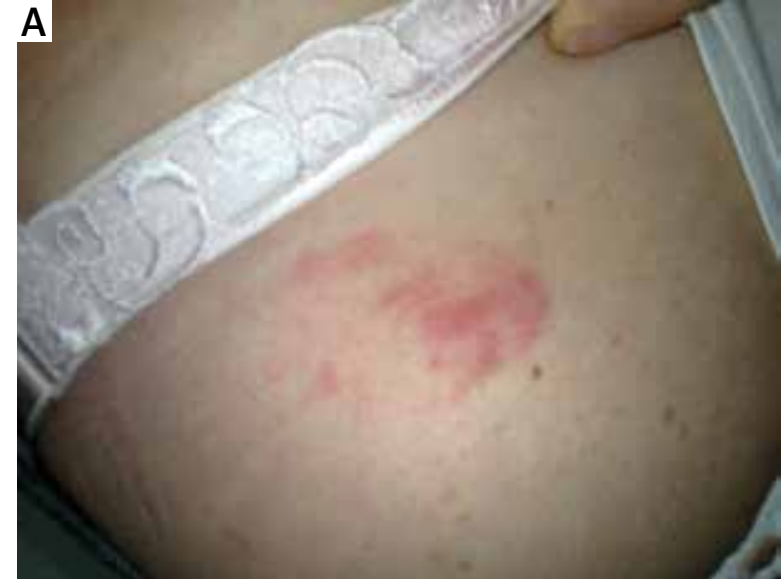

C

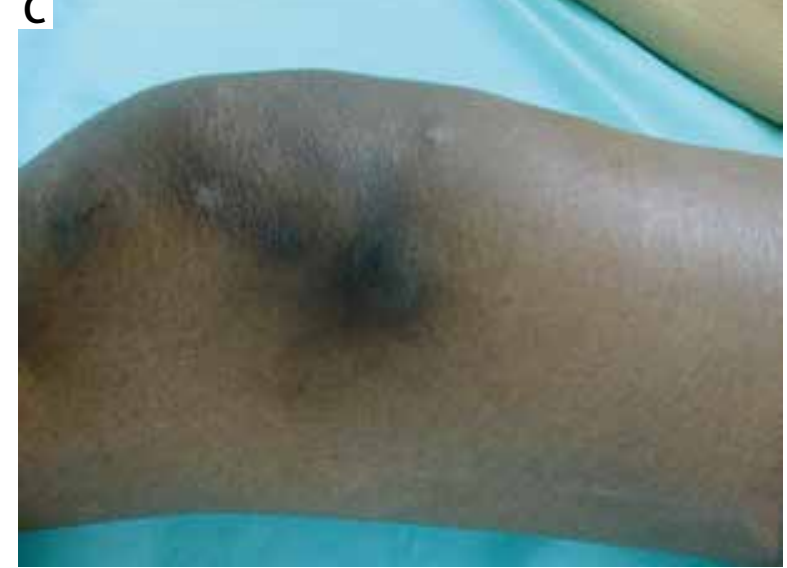

B

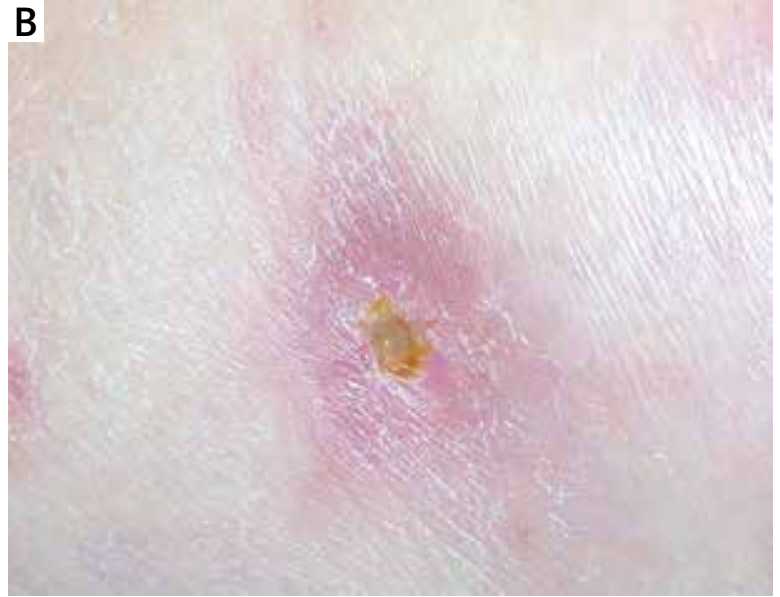

Fig. 3. Draining sinus four weeks after ${ }^{90} \mathrm{Y}$ RSV (A, B). Formation of scar tissue six months after RSV (C).
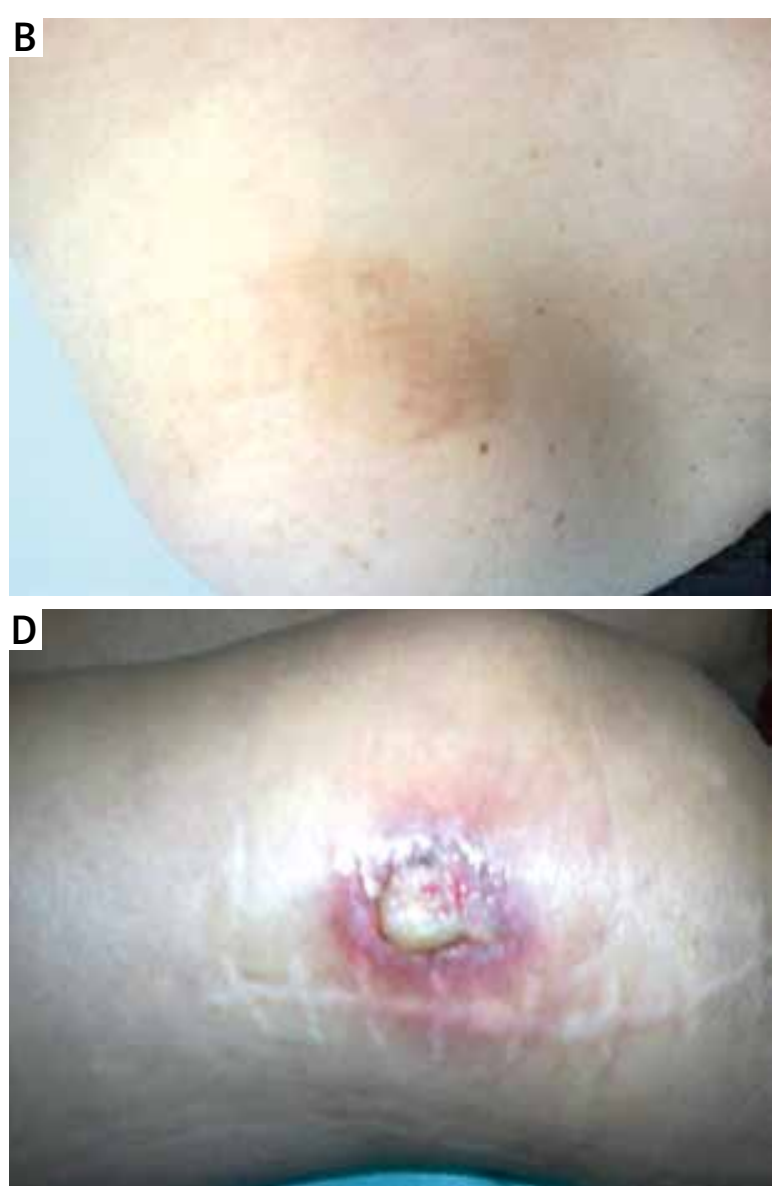

Fig. 4. Beta burns one week (A) and two weeks (B) after shoulder joint RSV with ${ }^{186}$ Re. Injection site skin discoloration one year after ${ }^{90} \mathrm{Y}$ knee RSV (C). Superficial skin necrosis six weeks after ${ }^{90} \mathrm{Y}$ knee RSV (D). 

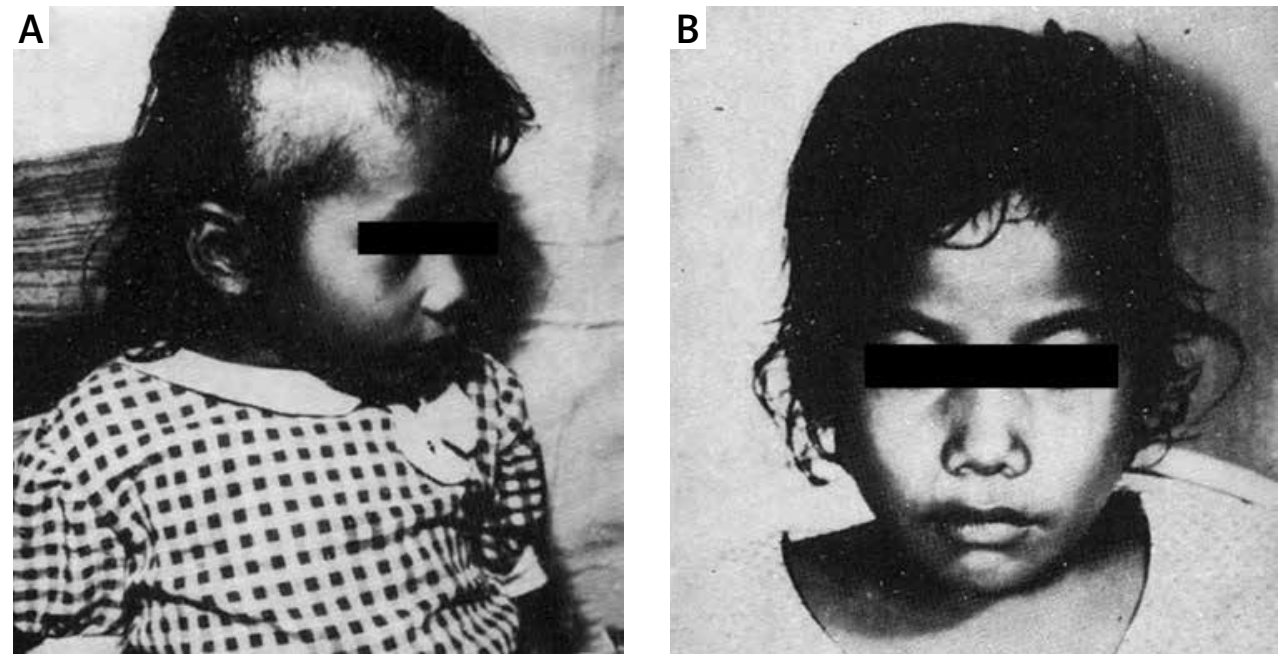

Fig. 5. Hair loss in course of beta burns 14 days after exposure to nuclear fallout (A). Complete hair regrowth after six months (B) [12]. (This image is a work of a United States Department of Energy [or predecessor organization] employee, taken or made as part of that person's official duties. As a work of the U.S. federal government, the image is in the public domain.)

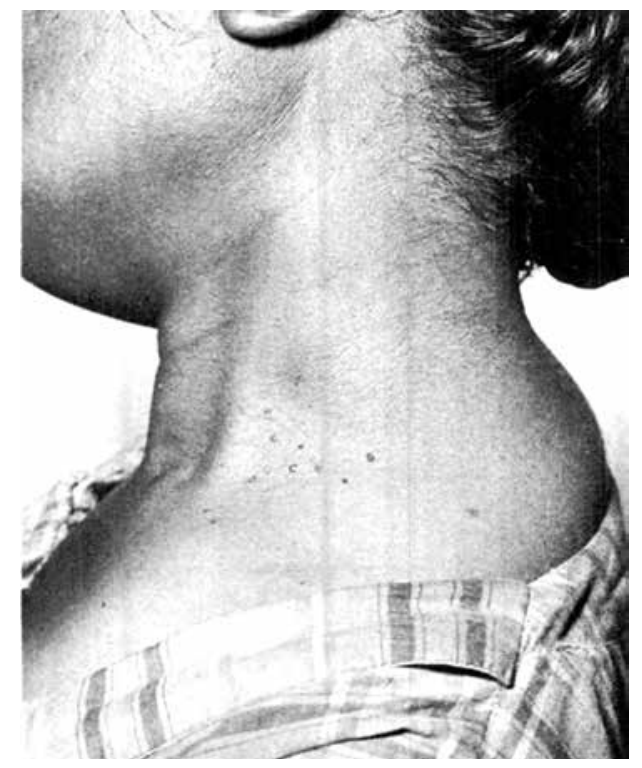

Fig. 6. Residual pigmented lesions seven years after nuclear fallout beta burn [13]. (This image is a work of a United States Department of Energy [or predecessor organization] employee, taken or made as part of that person's official duties. As a work of the U.S. federal government, the image is in the public domain.)

The closest approximation of RSV-related beta burns was observed following detonation of the Castle Bravo thermonuclear device in 1954 on Bikini atoll, when high winds carried nuclear fallout to inhabited parts of the Marshall Islands and a Japanese fishing boat, Daigo Fukuryū Maru. The 239 Marshallese islanders and 23 Japanese fishermen were far enough from the explosion not to be affected by either fireball, shockwave or blast-related gamma radiation. Still, they were subjected to a fallout of pulverized coral dust mixed with various radioactive isotopes, including potent $\beta$-emitters. Skin exposure to these substances resulted in a plethora of lesions, ranging from sheer discoloration to painful seeping wounds. The affected islanders were under close medical supervision for years after the incident, so the natural history of their beta burns is well documented [10-13]. Most of the mild to moderate lesions healed between a few weeks and six months, with return of normal skin color and hair regrowth, while more severe burns left permanent discoloration or scarring (Fig. 5) [12].

Only a few burns were secondarily infected, while none required major surgery. During the years following the exposure, there was an increased incidence of pigmented maculae and nevi (Fig. 6) [13], but histopathological examinations proved them to be benign [10, 11].

These phenomena are reflected in the range and severity of beta burns observed after RSV, as can be seen in the two cases described above. Considering RSV complications, the most important lesson from the Castle Bravo incident is that even after fifteen years, none of the affected islanders developed radiation-related skin cancer or malignant melanoma [11]. While this should be confirmed in prospective studies, for the time being it seems reasonable to infer that beta burns after RSV carry little or no risk of malignant transformation. 


\section{Conclusions}

Although radiation necrosis after joint radioisotope therapy is uncommon, occurrence of low grade beta burns is possible even with proper injection technique. Should such lesions occur, a watchful waiting strategy seems advisable, since most beta burns will resolve without any intervention and probably pose no longterm risk.

The authors declare no conflicts of interest.

\section{References}

1. Chojnowski MM, Felis-Giemza A, Kobylecka M. Radionuclide synovectomy - essentials for rheumatologists. Reumatologia 2016; 54: 108-116.

2. Oztürk H, Oztemür Z, Bulut O. Treatment of skin necrosis after radiation synovectomy with yttrium-90: a case report. Rheumatol Int 2008; 28: 1067-1068.

3. García-Colmenero L, Martin-Ezquerra G, Monfort J, et al. Persistent cutaneous ulcers after Yttrium-90 synovectomy, an unusual complication: two case reports and a review of the literature. Int Wound I 2017; 14: 508-511.

4. Kampen WU, Matis E, Czech N, et al. Serious complications after radiosynoviorthesis. Survey on frequency and treatment modalities. Nuklearmedizin 2006; 45: 262-268.

5. Ahmada I, Nisar H. Dosimetry perspectives in radiation synovectomy. Physica Medica 2018; 47: 64-72.
6. Chojnowski MM, Kobylecka M, Płazińska MT, et al. Popliteal cyst after yttrium-90 radiosynovectomy-usefulness of delayed PET/CT imaging. Rheumatology (Oxford) 2014; 53: 1983.

7. Liepe K. Radiosynovectomy in the Therapeutic Management of Arthritis. World I Nucl Med 2015; 14: 10-15.

8. Borab Z, Mirmanesh MD, Gantz M, et al. Systematic review of hyperbaric oxygen therapy for the treatment of radiationinduced skin necrosis. J Plast Reconstr Aesthet Surg 2017; 70: 529-538.

9. Vanhavere F, Huyskens D, Struelens L. Peripheral neutron and gamma doses in radiotherapy with an $18 \mathrm{MV}$ linear accelerator. Radiat Prot Dosimetry 2004; 110: 607-612.

10. Conard RA, Meyer LM, Sutow WW, et al. Medical Survey of the People of Rongelap and Utirik Islands Nine and Ten Years After Exposure to Fallout Radiation. Upton, N.Y.: Brookhaven National Laboratory 1965.

11. Conard RA, Sutow WW, Lowrey A, et al. Medical survey of the people of Rongelap and Utirik islands thirteen, fourteen, and fifteen years after exposure to fallout radiation. Upton, N.Y.: Brookhaven National Laboratory 1970.

12. United States. Congress. Joint Committee on Atomic Energy. Biological and environmental effects of nuclear war: Hearing before the Special Subcommittee on Radiation of the Joint Committee on Atomic Energy, Congress of the United States, Eighty-sixth Congress, first session. Washington 1959.

13. Conard RA, MacDonald HE, Meyer LM, et al. Medical survey of Rongelap people seven years after exposure to fallout. Upton, N.Y.: Brookhaven National Laboratory 1962. 